\title{
Existence of Localized Solutions for a Classical Nonlinear Dirac Field
}

\author{
Thierry Cazenave ${ }^{1}$ and Luis Vazquez ${ }^{2}$ \\ 1 Analyse Numérique, Université Pierre et Marie Curie, 4, place Jussieu, F-75230 Paris Cedex 05, France \\ 2 Departamento de Fisica Teorica, Facultad de Ciencias Fisicas, Universidad Complutense, S-28040 \\ Madrid, Spain
}

\begin{abstract}
We prove the existence of stationary states for nonlinear Dirac equations of the form:

$$
i \gamma^{\mu} \partial_{\mu} \psi-m \psi+F(\bar{\psi} \psi) \psi=0 .
$$

We seek solutions which are separable in spherical coordinates and we use a shooting method for solving the associated problem of ordinary differential equations.
\end{abstract}

\section{Introduction}

In this paper we prove the existence of stationary states for nonlinear Dirac equations of the form

$$
i \sum_{\mu=0}^{3} \gamma^{\mu} \partial_{\mu} \psi-m \psi+F(\psi \psi) \psi=0
$$

under certain hypothesis on $F$.

The notation is the following: $\psi$ is defined on $\mathbb{R}^{4}$ with values in $\mathbb{C}^{4}, \partial_{\mu}=\partial / \partial x_{\mu}, m$ is a positive constant, $\gamma^{\mu}$ are $4 \times 4$ matrices given by

$$
\begin{array}{ll}
\gamma^{0}=\left(\begin{array}{rr}
I & 0 \\
0 & -1
\end{array}\right), & \gamma^{k}=\left(\begin{array}{rr}
0 & \sigma^{k} \\
-\sigma^{k} & 0
\end{array}\right) \quad k=1,2,3, \text { where } \\
\sigma^{1}=\left(\begin{array}{ll}
0 & 1 \\
1 & 0
\end{array}\right), & \sigma^{2}=\left(\begin{array}{rr}
0 & -i \\
i & 0
\end{array}\right), \quad \sigma^{3}=\left(\begin{array}{rr}
1 & 0 \\
0 & -1
\end{array}\right),
\end{array}
$$

and $\psi \psi=\left(\gamma^{0} \psi, \psi\right)$, where (,) is the usual scalar product in $\mathbb{C}^{4}$.

Nonlinear spinor fields giving rise to equations of the form (1.1) have been considered first by D. Ivanenko [7], H. Weyl [22], and by W. Heisenberg [6] in his unified theory of elementary particles. Later R. Finkelstein, C. F. Fronsdal and P. Kaus [4] considered the case of a spinor field with several types of fourth order self couplings. But it was M. Soler [16] who was the first to investigate the stationary 
states of the nonlinear Dirac field with the scalar fourth order self coupling (corresponding to $F(x)=x$ in (1.1)) proposing them as a model of elementary extended fermions. Subsequently, the electromagnetic interaction was introduced $[17,13,14]$ in order to construct a model of extended charged fermion, which in spite of its simplicity describes with a reasonable accuracy the properties of the nucleons [12]. To improve the model, the pseudoscalar fields were introduced in order to represent the cloud of pions $[15,5]$. A summary of the above models, with the numerical computations and further developments are described by Rañada $[10,11]$. Also the case $F(x)=x$ was considered by Rafelski [9], Takahashi [18] and Van der Merwe [23].

We are interested in stationary states, or localized solutions of (1.1), that is solutions of the form $\psi(t, x)=\exp (-i \omega t) \varphi(x)$ with $x=\left(x_{1}, x_{2}, x_{3}\right), t=x_{0}$. Furthermore we want $\varphi$ to be square integrable. Clearly, the equation for $\varphi: \mathbb{R}^{3} \rightarrow \mathbb{C}^{4}$ is:

$$
i \sum_{k=1}^{3} \gamma^{k} \partial_{k} \varphi-m \varphi+\omega \gamma^{0} \varphi+F(\bar{\varphi} \varphi) \varphi=0
$$

We shall prove the following result:

Theorem 1.1. Assume that $F:[0,+\infty] \rightarrow[0,+\infty]$ is a $C^{1}$ function satisfying $F(0)=0, F^{\prime}(x)>0$ for $x>0$ and $\lim _{x \rightarrow+\infty} F(x)=+\infty$. Then for any $\omega \in(0, m)$ there is $\varphi \in C^{1}\left(\mathbb{R}^{3}, \mathbb{C}^{4}\right)$ such that:

(i) $\bar{\varphi} \varphi(x)>0$ for $x \in \mathbb{R}^{3}$,

(ii) $\varphi$ and $\nabla \varphi$ have an exponential fall-off as $|x| \rightarrow+\infty$,

(iii) $\varphi$ satisfies $E q$. (1.2).

Following M. Wakano [20] and M. Soler [16] we seek solutions which are separable in spherical coordinates,

$$
\varphi(x)=\left(\begin{array}{l}
g(r)\left(\begin{array}{l}
1 \\
0
\end{array}\right) \\
i f(r)\left(\begin{array}{c}
\cos \theta \\
\sin \theta e^{i \varphi}
\end{array}\right)
\end{array}\right),
$$

where $f$ and $g$ are real valued radial functions.

The equations for $f$ and $g$ are (compare $[16,20]$ )

$$
\begin{aligned}
f^{\prime}+\frac{2}{r} f & =g\left(F\left(g^{2}-f^{2}\right)-(m-\omega)\right) \\
g^{\prime} & =f\left(F\left(g^{2}-f^{2}\right)-(m+\omega)\right) .
\end{aligned}
$$

We assume $f(0)=0$ in order to eliminate solutions with singularities at the origin. Therefore, for any given value of $g(0)$ there is a local solution of (1.3).

The difficulty comes from the fact that we want the corresponding solution $\varphi$ of (1.2) to be square integrable. Therefore we want a solution of (1.3) which exists globally and which goes to $(0,0)$ as $r \rightarrow+\infty$.

However the numerical study of (1.3) shows, at least for certain $F$ (compare 
$[10,11])$ that "most" of the solutions of $(1.3)$ either blow-up or converge to ( 0 , $\left.\pm\left(F^{-1}(m-\omega)\right)^{1 / 2}\right)$ as $r \rightarrow+\infty$, those going to $(0,0)$ being exceptional. Therefore we have to solve a shooting problem for (1.3). Our main result is the following:

Theorem 1.2. Assume that $F$ satisfies the hypothesis of Theorem 1.1 and that $\omega \in(0, m)$. Then there is $(f, g) \in C^{1}\left(\mathbb{R}_{+}, \mathbb{R}^{2}\right)$ such that

(i) $0<f(r) \leqq g(r) \leqq$ Cte $\exp \left(-\frac{m-\omega}{2} r\right)$ for $r>0$,

(ii) $(f, g)$ is a solution of $(1.3)$.

It is immediate that Theorem 1.1 is a consequence of Theorem 1.2. Theorem 1.2 will be proved in a slightly more general form in Sect. 3 (see Theorem 3.1 and Remark 3.2).

Our argument is in a way similar to the one used by H. Berestycki, P. L. Lions and L. A. Peletier [2] for a second order equation, with the additional difficulty that we have to deal with a system and that solutions of (1.3) are not a priori bounded.

The paper is organized as follows:

- In Sect. 2 we establish preliminary results concerning systems like (1.3). Some of those results rely on sharp estimates related to the particular structure of (1.3) and their proofs are somewhat technical.

- In Sect. 3, we state and prove our main result.

- Section 4 is devoted to further remarks and comments.

\section{Analysis of a System of O.D.E.'s}

Throughout Sects. 2 and 3 we shall make the following assumptions and notations:

$-m$ and $\omega$ are real numbers such that $0<\omega<m$.

$-\alpha$ is a real number, $\alpha>0$.

$-g \in C^{1}(\mathbb{R}, \mathbb{R})$ is an increasing function satisfying

$$
g(0)=0, \lim _{x \rightarrow+\infty} g(x)>m+\omega \quad \text { and } \quad g^{\prime}\left(g^{-1}(m-\omega)\right)>0 .
$$

- For convenience we define $l=\left(g^{-1}(m-\omega)\right)^{1 / 2}$.

-We also define the functions $G$ and $H$ by

$$
\begin{aligned}
G(x) & =\int_{0}^{x} g(s) d s, \quad x \in \mathbb{R}, \\
H(u, v) & =\frac{1}{2}\left[G\left(v^{2}-u^{2}\right)-m\left(v^{2}-u^{2}\right)+\omega\left(v^{2}+u^{2}\right)\right] \text { for }(u, v) \in \mathbb{R}^{2} .
\end{aligned}
$$

We shall study the system:

$$
\begin{aligned}
u^{\prime}+\frac{\alpha}{r} u & =v\left(g\left(v^{2}-u^{2}\right)-(m-\omega)\right) \\
v^{\prime} & =u\left(g\left(v^{2}-u^{2}\right)-(m+\omega)\right) .
\end{aligned}
$$

First we consider the associated conservative system:

$$
\begin{aligned}
& u^{\prime}=v\left(g\left(v^{2}-u^{2}\right)-(m-\omega)\right) \\
& v^{\prime}=u\left(g\left(v^{2}-u^{2}\right)-(m+\omega)\right) .
\end{aligned}
$$


Clearly (2.2) is the Hamiltonian system associated with $H$. We begin with:

Lemma 2.1. $H$ has the following properties:

(i) $\nabla H(u, v)=0$ if and only if $(u, v)=(0,0)$ or $(u, v)=(0, \pm l)$.

(ii) $H(u, v) \rightarrow+\infty,|u|+|v| \rightarrow+\infty$.

(iii) The minimum of $H$ is negative and is achieved for $(u, v)=(0, \pm l)$.

Proof.

(i) is obvious.

(ii) Let $x_{0}>0$ and $\varepsilon>0$ be such that $g\left(x_{0}\right)=m-\omega+\varepsilon$. Let $x \geqq x_{0}$. Then:

$$
G(x)-m x=\int_{0}^{x_{0}}(g(s)-m) d s+\int_{x_{0}}^{x}(g(s)-m) d s \geqq(\varepsilon-\omega) x+(\omega-m-\varepsilon) x_{0} .
$$

For $x \in\left[0, x_{0}\right]$ we have $G(x)-m x \geqq-m x$, and for $x \leqq 0$ we have $G(x)-m x \geqq 0$. Therefore there is $C$ such that $G(x)-m x \geqq(\varepsilon-\omega) x-C$ for $x \in \mathbb{R}$. Then $H(u, v) \geqq$ $\varepsilon\left(u^{2}+v^{2}\right)-C$; hence (ii).

(iii) Applying (i), (ii) we only have to check that $\operatorname{Min} H(u, v)<0$. Actually we shall prove the stronger property:

$$
\text { Let } 0<v \leqq l ; \text { then } H(0, v)<0 .
$$

Indeed, $H(0, v)=\frac{1}{2} \int_{0}^{v^{2}}(g(s)-(m-\omega)) d s$. However, for $0 \leqq s<l^{2}$ we have $g(s)-$ $(m-\omega)<0$; hence $(2.3)$.

Lemma 2.2. Let $\left(u_{0}, v_{0}\right) \in \mathbb{R} \times \mathbb{R}$. If $H\left(u_{0}, v_{0}\right) \neq 0$ and $H\left(u_{0}, v_{0}\right) \neq H(0, l)$, then the solution $(u, v)$ of $(2.2)$ with initial data $\left(u_{0}, v_{0}\right)$ is periodic.

Proof. Consider $\Gamma=\left\{(u, v), H(u, v)=H\left(u_{0}, v_{0}\right)\right\}$. It follows from Lemma 2.1 that $\Gamma$ is compact and that $|\nabla H| \geqq \alpha>0$ on $\Gamma$ for some $\alpha>0$. Therefore $\Gamma$ is a curve and $(u, v)$ must cover the whole of the connected component of $\Gamma$ containing $\left(u_{0}, v_{0}\right)$ in a finite time. Therefore, $(u, v)$ is periodic.

Lemma 2.3. Let $\left(u_{0}, v_{0}\right) \in \mathbb{R}^{2}$ and let $(u, v)$ be the solution of (2.2) with initial data $\left(u_{0}, v_{0}\right)$. Then we have

$$
\begin{aligned}
& \text { If there is } k>0 \text { such that } v(r) \equiv k \text { on some interval, } \\
& \text { then }(u(r), v(r)) \equiv(0, l) \text { on } \mathbb{R} \text {. } \\
& \text { If } 0<u_{0} \leqq v_{0} \text { and } H\left(u_{0}, v_{0}\right)>0 \text {, then there is } r_{0}>0 \\
& \text { such that } \operatorname{Inf}_{r \in\left[0, r_{0}\right]} u(r)>0, \underset{r \in\left[0, r_{0}\right]}{\operatorname{Inf} v(r)>0 \text { and } v\left(r_{0}\right)<u\left(r_{0}\right) \text {. }}
\end{aligned}
$$

Proof. If $v^{\prime}$ vanishes of some interval, then considering the second equation of (2.2) we see that $u^{\prime}$ also vanishes on the same interval. Hence (2.4) applying (i) of Lemma 2.1.

For proving (2.5) consider $r_{1}=\operatorname{Sup}\{r \geqq 0, u>0$ and $v>0$ on $[0, r]\}$. We shall first establish that $r_{1}<\infty$.

Indeed, if $r_{1}=+\infty$, then since $(u, v)$ is periodic there is $\delta>0$ such that $u(r) v(r) \geqq \delta$ for $r \geqq 0$. 
Consider now $h(r)=\frac{1}{2}\left(u^{2}(r)-v^{2}(r)\right)$.

From (2.2) we obtain $h^{\prime}(r)=2 \omega u(r) v(r) \geqq 2 \omega \delta>0$.

This is a contradiction since $h$ is periodic. Therefore $r_{1}<\infty$. Assume now that $v\left(r_{1}\right)>0$. Then $u\left(r_{1}\right)=0$ and $u^{\prime}\left(r_{1}\right) \leqq 0$. From the first equation of (2.2) we get $g\left(v^{2}\left(r_{1}\right)\right)$ $-(m-\omega) \leqq 0$, hence $0<v\left(r_{1}\right) \leqq l$.

On applying (2.3) we obtain that $H\left(u\left(r_{1}\right), v\left(r_{1}\right)\right)<0$, which is a contradiction. Hence $v\left(r_{1}\right)=0$ and $u\left(r_{1}\right)>0$. Therefore, on choosing $r_{0}$ close enough to $r_{1}$ we obtain (2.5).

We shall now study the system (2.1). We begin with:

Lemma 2.4. Let $x \in \mathbb{R}$. Then there is $\tau>0$ and there is $\left(u_{x}, v_{x}\right) \in C^{1}\left([0, \tau], \mathbb{R}^{2}\right)$, unique solution of $(2.1)$ satisfying $u_{x}(0)=0, v_{x}(0)=x$. In addition, $\left(u_{x}, v_{x}\right)$ can be extended on a maximal interval $\left[0, R_{x}\left[\right.\right.$ with either $R_{x}=+\infty$ or $R_{x}<\infty$ and $\lim _{r \rightarrow R_{x}}\left|u_{x}\right|+\left|v_{x}\right|=+\infty$.

Furthermore, $\left(u_{x}, v_{x}\right)$ depend continuously on $x$, uniformly on $[0, R]$ for any $R<R_{x}$. Proof. We just write (2.1) in the form:

$$
\begin{aligned}
& u(r)=\frac{1}{r^{\alpha}} \int_{0}^{r} s^{\alpha} v(s)\left(g\left(v^{2}(s)-u^{2}(s)\right)-(m-\omega)\right) d s \\
& v(r)=x+\int_{0}^{r} u(s)\left(g\left(v^{2}(s)-u^{2}(s)\right)-(m+\omega)\right) d s .
\end{aligned}
$$

Since the right-hand side of $(2.1)$ is a Lipschitz continuous function of $(u, v)$ we may use a classical contraction mapping argument.

Next, we prove the following perturbation result:

Lemma 2.5. Let $\left(u_{0}, v_{0}\right) \in \mathbb{R}^{2}$ and let $(u, v)$ be the solution of (2.2) with initial data $\left(u_{0}, v_{0}\right)$. Let $\left(u_{n}^{0}, v_{n}^{0}\right)$ and $\rho_{n}$ be such that

$$
\underset{n \rightarrow \infty}{\rho_{n} \rightarrow+\infty} \text { and }\left(u_{n}^{0}, v_{n}^{0}\right) \underset{n \rightarrow \infty}{\rightarrow}\left(u_{0}, v_{0}\right) .
$$

Let $\left(u_{n}, v_{n}\right)$ be a solution of

$$
\begin{aligned}
u_{n}^{\prime}+\frac{\alpha}{\rho_{n}+r} u_{n} & =v_{n}\left(g\left(v_{n}^{2}-u_{n}^{2}\right)-(m-\omega)\right) \\
v_{n}^{\prime} & =u_{n}\left(g\left(v_{n}^{2}-u_{n}^{2}\right)-(m+\omega)\right)
\end{aligned}
$$

such that $u_{n}(0)=u_{n}^{0}, v_{n}(0)=v_{n}^{0}$, and let $\left[0, \tau_{n}\right)$ be the maximal existence interval of $\left(u_{n}, v_{n}\right)$. Then

(i) $\lim _{n \rightarrow \infty} \tau_{n}=+\infty$,

(ii) $\left(u_{n}, v_{n}\right)$ converges to $(u, v)$ uniformly on bounded intervals.

Proof. We have to prove that for any $R<\infty$ we have $\lim _{n \rightarrow \infty} \tau_{n}>R$ and $\left(u_{n}, v_{n}\right)$ converges to $(u, v)$ uniformly on $[0, R]$. Then, consider $R<\infty$. Since $\bigcup_{r \in[0, R]}\{(u(r), v(r))\}$ is a bounded set we obtain on applying a contraction argument 
that there exist $\delta>0$ and $\tau>0$ with the following property: For any $\rho \geqq 1, r_{0} \in[0, R]$ and $\left(w_{0}, z_{0}\right) \in \mathbb{R}^{2}$ such that $\left|w_{0}-u\left(r_{0}\right)\right|+\left|z_{0}-u\left(r_{0}\right)\right| \leqq \delta$, there is a solution $(w, z) \in C^{1}\left([0, \tau], \mathbb{R}^{2}\right)$ of:

$$
\begin{aligned}
w^{\prime}+\frac{\alpha}{\rho+r} w & =z\left(g\left(z^{2}-w^{2}\right)-(m-\omega)\right) \\
z^{\prime} & =w\left(g\left(z^{2}-w^{2}\right)-(m-\omega)\right)
\end{aligned}
$$

with $w(0)=w_{0}, z(0)=z_{0}$ and $\operatorname{Sup}_{r \in[0, \tau]}|w(r)|+|z(r)| \leqq 2 M$, where $M=\operatorname{Sup}_{r \in[0, R]}|u(r)|+$ $|v(r)|$.

Let $K$ be the Lipschitz constant of the second line of (2.6) on the ball of radius $2 M$ or $\mathbb{R}^{2}$. Then we get from (2.6), (2.2):

$$
\begin{gathered}
\left|w(r)-u\left(r+r_{0}\right)\right|+\left|z(r)-v\left(r+r_{0}\right)\right| \leqq\left|w_{0}-u\left(r_{0}\right)\right|+\mid\left(z_{0}-v\left(r_{0}\right) \mid\right. \\
+\int_{0}^{r} \frac{\alpha}{s+r_{0}}|w(s)| d s+\int_{0}^{r} K\left(\left|w(s)-u\left(s+r_{0}\right)\right|+\left|z(s)-v\left(r+r_{0}\right)\right|\right) d s .
\end{gathered}
$$

Applying Gronwall's Lemma we obtain:

$$
\begin{aligned}
& \left|w(r)-u\left(r+r_{0}\right)\right|+\mid\left(z(r)-v\left(r+r_{0}\right) \mid\right. \\
& \quad \leqq\left(\left|w_{0}-u\left(r_{0}\right)\right|+\mid\left(z_{0}-v\left(r_{0}\right) \mid+\frac{2 M \alpha \tau}{\rho}\right) \exp (K \tau) \text { for } 0 \leqq r \leqq \tau .\right.
\end{aligned}
$$

Applying this with $\rho=\rho_{n}, r_{0}=0, w_{0}=u_{n}^{0}, z_{0}=v_{n}^{0}$ we obtain that $\lim \tau_{n} \geqq \tau$ and $\left(u_{n}, v_{n}\right)$ converges uniformly to $(u, v)$ on $(0, \tau)$.

Iterating this argument $n$ times with $n \tau \leqq R \leqq(n+1) \tau$ we obtain Lemma 2.5.

Lemma 2.6. Let $x \in \mathbb{R}$. Then for any $r \in\left[0, R_{x}\right)$ we have

$$
\frac{d}{d r} H\left(u_{x}(r), v_{x}(r)\right)=\frac{\alpha}{r} u_{x}^{2}(r)\left(g\left(v_{x}^{2}(r)-u_{x}^{2}(r)\right)-(m+\omega)\right) .
$$

Proof. Equation (2.7) is derived from elementary computations.

Lemma 2.7. Let $x>0$ be such that $m-\omega \leqq g\left(x^{2}\right) \leqq m+\omega$. Then $R_{x}=+\infty$ and $H\left(u_{x}(r), v_{x}(r)\right)<H(0, x)$ for $r>0$.

Proof. Let $(u, v)$ be such that $g\left(v^{2}-u^{2}\right)>m+\omega$. Then

$$
H(u, v)=H(0, x)+(1 / 2) \int_{x^{2}}^{v^{2}-u^{2}}(g(s)-m) d s+\omega\left(v^{2}+u^{2}-x^{2}\right)>H(0, x)+\omega u^{2} .
$$

Therefore the sets $\{(u, v), H(u, v) \leqq H(0, x)\}$ and $\left\{(u, v), g\left(v^{2}-u^{2}\right)-(m+\omega)>0\right\}$ are disconnected.

Then the lemma follows from (2.7) and an obvious continuity argument.

Lemma 2.8. Let $x>0$ and $\rho \in\left(0, R_{x}\right)$ be such that $u_{x}(r)>0, v_{x}(r)>0$ for $0<r<\rho$.

Assume that there is $\theta \in(0, \rho)$ such that $u_{x}(\theta)-v_{x}(\theta)=\delta>0$. Then $u_{x}(r)-v_{x}(r) \geqq \delta$ for $r \in[\theta, \rho]$.

Proof. Let $x$ be as above and let $h(r)=v_{x}(r)-u_{x}(r)$ for $0 \leqq r \leqq \rho$. We have $h(0)>0$ 
and $h(\theta)<0$. Therefore there is $\beta \in(0, \theta)$ such that $h(\beta)=0$ and $h^{\prime}(\beta) \leqq 0$.

From (2.1) we obtain immediately $\beta \geqq \alpha / 2 \omega$, hence $\theta \geqq \alpha / 2 \omega$. Let now $r \in[\theta, \rho]$. We have

$$
h^{\prime}(r)=\left(m-\omega-g\left(v^{2}-u^{2}\right)\right) h+\left(\frac{\alpha}{r}-2 \omega\right) u \leqq\left(m-\omega-g\left(v^{2}-u^{2}\right)\right) h .
$$

Since $\left(m-\omega-g\left(v^{2}-u^{2}\right)\right) \geqq m-\omega>0$ when $h \leqq 0$, the above inequality yields $h^{\prime}(r) \leqq 0$ for $r \in[\theta, \rho]$. Hence the lemma.

Lemma 2.9. Let $x>0$ and $\rho \in\left(0, R_{x}\right)$ be such that $u_{x}(r)>0$ and $v_{x}(r)>0$ for $r \in(0, \rho)$.

Assume there is $\theta \in(0, \rho)$ such that $g\left(v_{x}^{2}(\theta)-u_{x}^{2}(\theta)\right)-(m+\omega)<0$, and let $\tau=\operatorname{Sup}\left\{r \in[0, \rho], \quad g\left(v_{x}^{2}-u_{x}^{2}\right)-(m+\omega) \geqq 0 \quad\right.$ on $\left.[0, r]\right\}$. Then we have $\tau \geqq(\alpha / 2 \omega)\left(u_{x}(\tau) / v_{x}(\tau)\right)$ and $g\left(v_{x}^{2}(r)-u_{x}^{2}(r)\right)-(m+\omega)<0$ for $r \in(\tau, \rho)$.

Proof. Suppose first that $g\left(x^{2}\right)-(m+\omega) \leqq 0$. Applying Lemma 2.7 we obtain that $H\left(u_{x}(r), v_{x}(r)\right)<H(0, x)$ for $r>0$.

As pointed out in the proof of Lemma 2.7, this implies that

$$
g\left(v_{x}^{2}(r)-u_{x}^{2}(r)\right)-(m+\omega)<0 \text { for } r>0 .
$$

Therefore we have $\tau=0$ and the conclusion of Lemma 2.9 holds.

Suppose now that $g\left(x^{2}\right)>(m+\omega)$. Then we have $\tau>0$. Consider $k(r)=$ $v_{x}^{2}(r)-u_{x}^{2}(r)$ for $r \in[0, \rho]$. From (2.1) we get:

$$
k^{\prime}(r)=2\left(\frac{\alpha}{r} u_{x}(r)-2 \omega v_{x}(r)\right) u_{x}(r)
$$

It follows from the definition of $\tau$ that $k^{\prime}(\tau) \leqq 0$, hence $\tau>(\alpha / 2 \omega)\left(u_{x}(\tau) / v_{x}(\tau)\right)$. It remains to prove that $g(k(r))-(m+\omega)<0$ for $r \in(\tau, \rho)$. We argue by contradiction. If this is wrong we can find $r_{0}$ and $r_{1}$ such that $\tau<r_{0}<r_{1}, g(k(r))-(m+\omega)<0$ for $r \in\left(r_{0}, r_{1}\right)$ and $g\left(k\left(r_{0}\right)\right)=g\left(k\left(r_{1}\right)\right)=m+\omega$.

However, we have $k^{\prime}\left(r_{0}\right) \leqq 0$ and $k^{\prime}\left(r_{1}\right) \geqq 0$. From (2.8) we obtain

$$
\frac{u_{x}\left(r_{0}\right)}{v_{x}\left(r_{0}\right)} \leqq \frac{u_{x}\left(r_{1}\right)}{v_{x}\left(r_{1}\right)} .
$$

Since $g\left(k\left(r_{0}\right)\right)=g\left(k\left(r_{1}\right)\right)=m+\omega$, this implies that $v_{x}\left(r_{1}\right) \geqq v_{x}\left(r_{0}\right)$.

On the other hand, considering (2.1) we see that $v_{x}^{\prime}<0$ for $r \in\left(r_{0}, r_{1}\right)$. Hence $v_{x}\left(r_{1}\right)<v_{x}\left(r_{0}\right)$ which is a contradiction. This ends the proof of Lemma 2.

Lemma 2.10. Let $x>0$ be such that $R_{x}=+\infty$ and

$$
0<u_{x}(r) \leqq v_{x}(r) \leqq \text { Cte for } r>0 .
$$

Then there is $C$ such that $v_{x}(r) \leqq C \exp \left(-\frac{m-\omega}{2} r\right)$.

Proof. Let us denote $\left(u_{x}, v_{x}\right)$ by $(u, v)$. Let $\tau=\operatorname{Sup}\left\{r \geqq 0, g\left(v^{2}-u^{2}\right)-(m+\omega) \geqq 0\right.$ on $[0, r]\}$. Considering $(2.1)$ we see that $v^{\prime} \geqq 0$ on $[0, \tau]$. Therefore $v(r) \geqq x$ for $r \in[0, \tau]$. 
We have then:

$$
u^{\prime}+\frac{\alpha}{r} u \geqq 2 \omega v \geqq 2 \omega x \text { on } \quad[0, \tau] .
$$

Hence $u(\tau) \geqq(2 \omega x /(\alpha+1)) \tau$. Since $u$ is bounded, $\tau$ is finite.

Applying Lemma 2.9 we have $g\left(v^{2}-u^{2}\right)-(m+\omega)<0$ on $(\tau,+\infty)$. Thus $v$ is decreasing on $(\tau,+\infty)$. Then there is $\delta \geqq 0$ such that $v(r) \searrow \delta$.

Assume for a moment that $\delta=0$. Then $(u, v) \underset{r \rightarrow \infty}{\rightarrow \rightarrow}(0,0)$ and $g\left(v^{2}-u^{2}\right) \underset{r \rightarrow \infty}{\rightarrow} 0$.

Considering (2.1) we have for $r$ large enough

$$
\begin{aligned}
& u^{\prime} \leqq-\frac{\alpha}{r} u-\frac{m-\omega}{2} v \\
& v^{\prime} \leqq-\frac{m+\omega}{2} u
\end{aligned}
$$

Hence: $(u+v)^{\prime}+((m-\omega) / 2)(u+v) \leqq 0$ for $r$ large.

Integrating the above equation we see that $(u, v)$ has the prescribed exponential decay. Therefore it only remains to prove that $\delta=0$. We argue by contradiction and we assume $\delta>0$.

Let $r_{n} \rightarrow+\infty$ be such that $u\left(r_{n}\right) \rightarrow k$.

Let $(w, z)$ be the solution of $(2.2)$ with initial data $(k, \delta)$. It follows from Lemma 2.5 that $\left(u\left(r_{n}+\cdot\right), v\left(r_{n}+\cdot\right)\right)$ converges to $(w, z)$ uniformly on bounded intervals.

Since $v\left(r_{n}+r\right) \rightarrow \delta$ for any $r>0$, we have $z(r) \equiv \delta$, hence from (2.4) we have $k=0$ and $\delta=l$.

This argument shows that indeed $(u, v) \rightarrow(0, l)$. We now put $v=l+w$. The equations for $(u, w)$ are:

$$
\begin{aligned}
u^{\prime}+\frac{\alpha}{r} u & =(l+w)\left(g\left(l^{2}+w^{2}+2 w l-u^{2}\right)-(m-\omega)\right) \\
w^{\prime} & =u\left(g\left(l^{2}+w^{2}+2 w l-u^{2}\right)-(m+\omega)\right) .
\end{aligned}
$$

Let us put $a=g^{\prime}\left(l^{2}\right)>0$. Since $g\left(l^{2}\right)=(m-\omega)$, we have

$$
g\left(l^{2}+w^{2}+2 w l-u^{2}\right)=(m-\omega)+2 l a w+o(u+w) .
$$

Therefore we have:

$$
\begin{aligned}
u^{\prime}+\frac{\alpha}{r} u & =2 l^{2} a w+o(u+w) \\
w^{\prime} & =-2 \omega u+o(u+w) .
\end{aligned}
$$

Hence: $(w-u)^{\prime}=\omega(w-u)-(\omega-(\alpha / r)) u-\left(\omega+2 l^{2} a\right) w+o(u+w)$.

For $r$ large we obtain $(w-u)^{\prime}-\omega(w-u) \leqq 0$. Hence $e^{-\omega r}(w-u)$ is nonincreasing for $r$ large. Since $e^{-\omega r}(w-u) \underset{r \rightarrow \infty}{\rightarrow 0}$ we must have $w-u \geqq 0$ for $r$ large.

Therefore we can replace $o(u+w)$ by $o(w)$.

From the first equation of (2.9) we obtain

$$
u^{\prime}+\frac{\alpha}{r} u \geqq l^{2} a w \text { for } r \text { large. }
$$


Hence $u^{\prime} \geqq\left(l^{2} a-\alpha / r\right) u$ for $r$ large. This implies $u(r) \geqq C e^{\left(l^{2} a / 2\right) r}$ for $r$ large which is a contradiction and Lemma 2.10 is proved.

\section{The Main Result}

We are now in a position to state and prove our main result. We assume that $\alpha, \omega, m$ and $g$ satisfy the same conditions as in Sect.2. Then we have:

Theorem 3.1. There is a solution $(u, v) \in C^{1}\left([0,+\infty), \mathbb{R}^{2}\right)$ of the system

$$
\begin{aligned}
u^{\prime}+\frac{\alpha}{r} u & =v\left(g\left(v^{2}-u^{2}\right)-(m-\omega)\right) \\
v^{\prime} & =u\left(g\left(v^{2}-u^{2}\right)-(m+\omega)\right)
\end{aligned}
$$

such that $u(0)=0$, and

$$
0<u(r) \leqq v(r) \leqq \text { Cte } \exp \left(-\frac{m-\omega}{2} r\right) \text { for } r>0 .
$$

Remark 3.2. Theorem 1.2 is a consequence of Theorem 3.1 above. Indeed, let $F$ satisfy the hypothesis of Theorem 1.2, and consider $g$ defined by:

$$
\begin{array}{ll}
g(x)=F(x) & \text { for } \quad x \geqq 0, \\
g(x)=-F(-x) & \text { for } \quad x \leqq 0 .
\end{array}
$$

Then $g$ satisfies the hypothesis of Theorem 3.1, and therefore Theorem 1.2 is proved. Proof of Theorem 3.1. The proofs proceeds in several steps. We define a set I as follows:

$$
I=\left\{x>l / \exists r_{x} \in\left(0, R_{x}\right), u_{x}>0 \text { and } v_{x}>0 \text { on }\left(0, r_{x}\right) \text { and } u_{x}\left(r_{x}\right)=0\right\} .
$$

In Step 1 we shall prove that $I$ is a non-empty open set. Then in Step 2 we shall establish estimates for solutions of (2.1) with initial data in $I$.

Finally, in Step 3 we shall prove that $\left(u_{\bar{x}}, v_{\bar{x}}\right)$ with $\bar{x}=\operatorname{Sup}$ I satisfies the conclusions of Theorem 3.1 .

Step 1. I is a non-empty open set.

We have $H(0, l)<0$ and $H(0, x) \underset{x \rightarrow+\infty}{\rightarrow}+\infty$ from Lemma 2.1 Thus there is $x_{0}>l$ such that $H\left(0, x_{0}\right)=0$. We put $x_{1}=\operatorname{Inf}\left(x_{0}, g^{-1}(m+\omega)\right)$. Then we claim that $\left(l, x_{1}\right) \subset I$. Indeed, let $x \in\left(l, x_{1}\right)$.

From Lemma 2.7, we have $R_{x}=+\infty$ and $H\left(u_{x}(r), v_{x}(r)\right)<H(0, x)$, for $r>0$. Since clearly $H(0, x)<0$, we have

$$
H\left(u_{x}(r), v_{x}(r)\right)<0 \quad \text { for } r>0
$$

On the other hand, a straightforward computation shows that $H(y, 0) \geqq 0$ for $y \in \mathbb{R}$. As a consequence $v_{x}(r)$ can not vanish in a finite time. Therefore, $v_{x}(r)>0$ for $r>0$. As well, we can check easily that $H(u, v) \geqq 0$ when $0 \leqq|v| \leqq|u|$. Therefore we have $\left|u_{x}(r)\right| \leqq v_{x}(r)$ for $r>0$. 
On the other hand we have $(\alpha+1) u_{x}^{\prime}(0)=x\left(g\left(x^{2}\right)-(m-\omega)\right)>0$. Hence $u_{x}(r)>0$ for $r>0$ and small.

Thus it only remains to prove that $u_{x}(r)$ vanishes for some $r>0$. We argue by contradiction and we assume that $u_{x}(r)>0$ for $r>0$. Since $H\left(u_{x}, v_{x}\right)$ is bounded, $\left(u_{x}, v_{x}\right)$ is bounded by Lemma 2.1 and we can apply Lemma 2.10 , which asserts that $\left(u_{x}, v_{x}\right) \underset{r \rightarrow \infty}{\rightarrow}(0,0)$. This implies $\lim _{r \rightarrow \infty} H\left(u_{x}, v_{x}\right)=0$, which is a contradiction since $H\left(u_{x}, v_{x}\right) \leqq H(0, x)<0$. Therefore $u_{x}$ has to vanish and $x \in I$.

The open character of $I$ is an immediate consequence of the continuous dependence of $\left(u_{x}, v_{x}\right)$ on $x$.

Step 2. Estimates for solutions of (2.1) with initial data in $I$. We begin with:

Lemma 3.3. Let $x \in I$. Then:

(i) $0<u_{x}(r) \leqq v_{x}(r)$ for $r \in\left(0, r_{x}\right)$

(ii) There is $\tau_{x} \in\left[0, r_{x}\right)$ such that $v_{x}(r) \leqq v_{x}\left(\tau_{x}\right)$ for $r \in\left[0, r_{x}\right)$.

\section{Proof.}

(i) We argue by contradiction and we assume that there is $r_{0} \in\left(0, r_{x}\right)$ such that $u_{x}\left(r_{0}\right)-v_{x}\left(r_{0}\right)=\delta>0$. Applying Lemma 2.8 we obtain that $u_{x}\left(r_{x}\right)-v_{x}\left(r_{x}\right) \geqq \delta$. This is impossible since this would imply $v_{x}\left(r_{x}\right)<0$. Thus (i) is proved.

(ii) Since $u_{x}\left(r_{x}\right)=0$, we have $v_{x}\left(r_{x}\right)>0$ because $(0,0)$ is an equilibrium point of $(2.1)$ and can not be reached in a finite time. On the other hand, we have $u_{x}^{\prime}\left(r_{x}\right) \leqq 0$, which implies $g\left(v_{x}^{2}\left(r_{x}\right)\right)-(m-\omega) \leqq 0$. Hence $g\left(v_{x}^{2}\left(r_{x}\right)\right)-(m+\omega)<0$.

On applying Lemma 2.9, we obtain the existence of $\tau_{x} \in\left[0, r_{x}\right)$ such that $g\left(v_{x}^{2}-u_{x}^{2}\right)-(m+\omega) \geqq 0$ on $\left[0, \tau_{x}\right)$ and $g\left(v_{x}^{2}-u_{x}^{2}\right)-(m+\omega)<0$ on $\left(\tau_{x}, r_{x}\right)$.

Therefore $v_{x}$ is non-decreasing on $\left(0, \tau_{x}\right)$ and decreasing on $\left(\tau_{x}, r_{x}\right)$, thus (ii) holds.

Then we have the following estimate:

Lemma 3.4. Let $M=\operatorname{Sup}_{x \in I} v_{x}\left(\tau_{x}\right)$. Then $M<+\infty$.

Proof. We use a contradiction argument and we assume that there is $x_{n} \in I$ such that $v_{x_{n}}\left(\tau_{x_{n}}\right) \underset{n \rightarrow \infty}{\rightarrow \infty} \infty$

For convenience we denote $u_{x_{n}}, v_{x_{n}}, \tau_{x_{n}}, r_{x_{n}}, R_{x_{n}}$ by $u_{n}, v_{n}, \tau_{n}, r_{n}, R_{n}$.

For $n$ large enough we must have $g\left(x_{n}^{2}\right)>(m+\omega)$, otherwise $v_{n}$ would be bounded by Lemmas 2.1 and 2.7. Therefore $\tau_{n}>0$ and $g\left(v_{n}^{2}\left(\tau_{n}\right)-u_{n}^{2}\left(\tau_{n}\right)\right)=(m+\omega)$. Thus we have:

$$
\operatorname{Lim}_{n \rightarrow \infty} \frac{u_{n}\left(\tau_{n}\right)}{v_{n}\left(\tau_{n}\right)}=1
$$

On applying Lemma 2.1 (ii) we obtain the existence of $k>0$ such that $H(k, v)>0$ for any $v \in \mathbb{R}$.

Since by (3.1) we have $u_{n}\left(\tau_{n}\right) \underset{n \rightarrow \infty}{\rightarrow} \infty$, and $u_{n}\left(r_{n}\right)=0$, for $n$ large enough there is $\rho_{n} \in\left(\tau_{n}, r_{n}\right)$ such that

$$
u_{n}(r)>k \quad \text { for } \quad r \in\left(\tau_{n}, \rho_{n}\right), \quad u_{n}\left(\rho_{n}\right)=k .
$$


From the definition of $\tau_{n}$ and from Lemma 3.3, (i) we have

Thus

$$
0 \leqq g\left(v_{n}^{2}(r)-u_{n}^{2}(r)\right) \leqq m+\omega \text { for } \quad r \in\left(\tau_{n}, r_{n}\right) .
$$

$$
\left|g\left(v_{n}^{2}(r)-u_{n}^{2}(r)\right)-(m-\omega)\right| \leqq 3 m \text { for } \quad r \in\left(\tau_{n}, r_{n}\right) .
$$

Furthermore, let $r \in\left(\tau_{n}, \rho_{n}\right)$. We have:

$$
v_{n}^{2}(r)-u_{n}^{2}(r) \leqq g^{-1}(m+\omega) .
$$

Thus:

$$
\begin{aligned}
v_{n}(r) & \leqq u_{n}(r)\left(1+\frac{g^{-1}(m+\omega)}{u_{n}^{2}(r)}\right)^{1 / 2} \\
& \leqq u_{n}(r)\left(1+\frac{g^{-1}(m+\omega)}{k^{2}}\right)^{1 / 2} \leqq C u_{n}(r)
\end{aligned}
$$

Considering (2.1) we obtain that

$$
\left|u_{n}^{\prime}+\frac{\alpha}{r} u_{n}\right| \leqq C u_{u} \quad \text { on } \quad\left(\tau_{n}, \rho_{n}\right) .
$$

In particular we have

Consider

$$
u_{n}^{\prime}+\frac{\alpha}{r} u_{n}+C u_{n} \geqq 0 \quad \text { on } \quad\left(\tau_{n}, \rho_{n}\right) .
$$

$$
\phi_{n}(r)=\log u_{n}(r)+C r+\alpha \log r .
$$

Then $\phi_{n}^{\prime} \geqq 0$ on $\left(\tau_{n}, \rho_{n}\right)$. Therefore $\phi_{n}\left(\rho_{n}\right) \geqq \phi_{n}\left(\tau_{n}\right)$. That is:

$$
C \rho_{n}+\log \rho_{n}^{\alpha} \geqq-\log k+\log u_{n}\left(\tau_{n}\right)+C \tau_{n}+\log \tau_{n}^{\alpha}
$$

From (3.1) and Lemma 2.9 we have $u_{n}\left(\tau_{n}\right) \underset{n \rightarrow \infty}{\rightarrow}+\infty$ and $\underline{\lim }_{n \rightarrow \infty} \tau_{n} \geqq \alpha / 2 \omega$. It follows easily that:

$$
\rho_{n} \underset{n \rightarrow+\infty}{\rightarrow}+\infty
$$

Let us set $\left(w_{n}, z_{n}\right)=\left(u_{n}\left(\rho_{n}+\cdot\right), v_{n}\left(\rho_{n}+\cdot\right)\right)$. We have $w_{n}(0)=k$ and by $(3.2) z_{n}(0)$ is bounded. Therefore we can assume, possibly by extracting a subsequence that

$$
z_{n}(0) \rightarrow \tilde{z} \geqq k .
$$

Let $(w, z)$ be the solution of $(2.8)$ with initial data $(k, \tilde{z})$. By definition of $k$, we have $H(k, \tilde{z})>0$.

On applying (2.5), we have the existence of $\gamma>0$ such that $\underset{r \in[0, \gamma]}{\operatorname{Inf}} w(r)>0, \underset{r \in[0, \gamma]}{\operatorname{Inf}}$ $z(r)>0$, and $z(\gamma)-w(\gamma)>0$.

On the other hand, on applying Lemma 2.5, and since $\rho_{n} \rightarrow \infty$, we obtain that $\left(w_{n}, z_{n}\right)$ converges to $(w, z)$ uniformly on $[0, \gamma]$. Therefore for $n$ large enough we have:

$$
\operatorname{Inf}_{r \in\left[0, \rho_{n}+\gamma\right]} u_{n}(r) \geqq 0, \quad \operatorname{Inf}_{r \in\left[0, \rho_{n}+\gamma\right]} v_{n}(r) \geqq 0
$$

and $u_{n}\left(\rho_{n}+\gamma\right)>v_{n}\left(\rho_{n}+\gamma\right)$. 
Therefore Lemma 3.3(i) provides a contradiction and Lemma 3.4 holds. $3^{\text {rd }}$ Step. Conclusion. From Lemmas 3.3 and 3.4 we have $\left.I \subset\right] l, M[$. Therefore we can put $\bar{x}=\operatorname{Sup} I<\infty$. Clearly $\bar{x} \notin I$. Let $(\bar{u}, \bar{v})=\left(u_{\bar{x}}, v_{\bar{x}}\right)$ and $\bar{R}=R_{\bar{x}}$.

On the other hand, since $\bar{x}>l$ we have $\bar{u}^{\prime}(0)>0$, and thus $\bar{u}>0$ and $\bar{v}>0$ for $r>0$ and small.

Since $\bar{x} \notin I, \bar{u}$ can not vanish before $\bar{v}$ does.

On the other hand, since $\bar{x} \in \bar{I}$, it follows from the continuous dependence of $\left(u_{x}, v_{x}\right)$ with respect to $x$ that $\bar{v}$ cannot vanish before $\bar{u}$. Since $(0,0)$ is a rest point of (2.1), $\bar{u}$ and $\bar{v}$ cannot vanish simultaneously.

Therefore $\bar{u}>0$ and $\bar{v}>0$ for $r \in(0, \bar{R})$.

Let $x_{n} \in I, x_{n} \rightarrow \bar{x}$. Let us denote $u_{x_{n}}, v_{x_{n}}, R_{x_{n}}, r_{x_{n}}$ by $u_{n}, v_{n}, R_{n}, r_{n}$. Let $\rho \in(0, \bar{R})$. Since $x_{n} \rightarrow \bar{x}$ we obtain that for $n$ large enough we have $R_{n}>\rho$ and that $\left(u_{n}, v_{n}\right)$ converges to $(\bar{u}, \bar{v})$ uniformly on $[0, \rho]$. Therefore we have $r_{n}>\rho$ for $n$ large and applying Lemma 3.4 we have

$$
\bar{u}(\rho)+\bar{v}(\rho) \leqq 2 M .
$$

Therefore $\bar{u}$ and $\bar{v}$ are bounded and $\bar{R}=+\infty$.

A similar argument shows that $0<\bar{u}(r) \leqq \bar{v}(r)$ for $r>0$. Then we can apply Lemma 2.10 and there is $C$ such that

$$
0<\bar{u}(r) \leqq \bar{v}(r) \leqq C \exp \left(-\frac{m-\omega}{2} r\right) .
$$

The proof of Theorem 3.1 is thereby complete.

\section{Further Remarks and Comments}

Remark 4.1. The Dirac equation (1.1) is in great contrast with the Klein-Gordon equation, since it possesses stationary states without restrictions on the growth of the nonlinear interaction $F$. (Compare [2] for the Klein-Gordon equation.)

Remark 4.2. We do not know if the condition $0<\omega<m$ is necessary in Theorem 1.1. On the other hand, this condition is almost necessary in Theorem 3.1. Indeed, it has been shown by L. Vazquez [19] that in the case $g(x)=x$, there is no solution of (2.1) such that the corresponding solution $\varphi$ of (1.2) is square integrable, in the case $|\omega|>m$. His argument works as well in the more general framework of Theorem 3.1.

In addition, elementary computations show that if $-m \leqq \omega \leqq 0$, there is no solution of (2.1) going to 0 as $r \rightarrow \infty$. This had already been observed on numerical experiments (compare [16]).

Considering again Eq. (1.2) it seems that no elementary transformation of the solution given by Theorem 3.1 can give a solution of (1.2) for $\omega>0$. For example, the charge conjugate of the solution given by Theorem 3.1 satisfies Eq. (1.2) with $\omega$ replaced by $-\omega$ but with $F(x)$ replaced by $F(-x)$.

Remark 4.3. Little seems to be known concerning the initial value problem (Cauchy problem) for Eq. (1.1). In particular we do not know if the stationary states are stable or not. For instance, some authors claim that the $(\bar{\psi} \psi)^{2}$ stationary states are unstable 
$[3,21,8]$ while others find regions of stable behaviour by using numerical computations [1]. Related to that question it would be of a great interest to solve directly Eq. (1.2) by a variational method, since better knowledge of the variational structure of the problem might give relevant information concerning the stability of stationary states. After the completion of this paper, the stability problem has been studied by Strauss and Vazquez [24].

\section{References}

1. Alvarez, A., Soler, M.: Energetic stability criterion for a nonlinear spinorial model. Phys. Rev. Lett. 50, 1230-1233 (1983)

2. Berestycki, H., Lions, P. L., Peletier, L. A.: Indiana Univ. Math. J. 30, 141-157 (1981)

3. Bogolubski, I. L.: On spinor soliton stability. Phys. Lett. A 73, 87-90 (1979)

4. Finkelstein, R., Fronsdal, C. F., Kaus, P.: Nonlinear spinor fields. Phys. Rev. 103, 1571-1579 (1956)

5. Garcia, L., Rañada, A. F.: A classical model of the nucleon. Prog. Theor. Phys. (Kyoto) 64, 671-693 (1980)

6. Heisenberg, W.: Doubts and hopes in quantumelectrodynamics. Physica 19, 897-908 (1953)

7. Ivanenko, D.: Sov. Phys. 13, 141-149 (1938)

8. Mathieu, P., Morris, T. F.: Instability of stationary states for nonlinear spinor models with quartic self-interaction. Phys. Lett. 126B, 74 (1983)

9. Rafelski, J.: Soliton solutions of a selfinteracting Dirac field in three space dimensions. Phys. Lett. B66, 262-266 (1977)

10. Rañada, A. F.: Classical nonlinear Dirac field models of extended particles. In: Quantum theory, groups, fields and particles. Barut, A. O. (ed.) Amsterdam: Reidel 1983

11. Rañada, A. F., Rañada, M. F.: Nonlinear model of $c$-number confined Dirac quarks. Phys. Rev. D29, 985-993 (1984)

12. Rañada, A. F., Ranada, M. F., Soler, M., Vazquez, L.: Classical electrodynamics of a nonlinear Dirac field with anomalous magnetic moment. Phys. Rev. D10, 517-525 (1976)

13. Rañada, A. F., Soler, M.: Perturbation theory for an exactly soluble spinor model in: interaction with its electromagnetic field. Phys. Rev. D8, 3430-3433 (1973)

14. Rañada, A. F., Uson, J., Vazquez, L.: Born-Infeld effects in the electromagnetic mass of an extended Dirac particle. Phys. Rev. D22, 2422-2424 (1980)

15. Rañada, A. F., Vazquez, L., Prog. Theor. Phys. (Kyoto) 56, 311-323 (1976)

16. Soler, M., Classical, stable, nonlinear spinor field with positive rest energy. Phys. Rev. D1, 2766-2769 (1970)

17. Soler, M.: Classical electrodynamics for a nonlinear spinorfield: Perturbative and exact approaches. Phys. Rev. 3424-3429 (1973)

18. Takahashi, K.: Soliton solutions of nonlinear Dirac equations. J. Math. Phys. 20, 1232-1238 (1979)

19. Vazquez, L.: Localized solutions of a non-linear spinor field. J. Phys. A10, 1361-1368 (1977)

20. Wakano, M.: Intensely localized solutions of the classical Dirac-Maxwell field equations. Prog. Theor. Phys. (Kyoto) 35, 1117-1141 (1966)

21. Werle, J.: Stability of particle-like solutions of non-linear Klein-Gordon and Dirac equations. Acta Physica Polonica B12, 601-616 (1981)

22. Weyl, H.: A remark on the coupling of gravitation and electron. Phys. Rev. 77, 699-701 (1950)

23. Van der Merwe, T.: Nuovo Cimento. 60A, 247 (1980)

24. Strauss, W. A., Vazquez, L.: On the stability under dilations of nonlinear spinor fields (submitted to Phys. Rev. D)

Communicated by L. Nirenberg

Received June 20, 1985 
